\title{
Task Specific Trajectory Profile Selection for Energy Efficient Servo Drive Movements
}

\author{
Christian Hansen, Kai Eggers, Jens Kotlarski, and Tobias Ortmaier \\ Institute of Mechatronic Systems, Gottfried Wilhelm Leibniz Universität Hannover, Hanover, Germany \\ E-mail: christian.hansen@imes.uni-hannover.de
}

\begin{abstract}
The energy demand of applications using inverter controlled electrical servo drives is considerably dependent on the applied trajectory profiles. In this paper, the improvement potential in supply energy consumption is analyzed with respect to the standard servo controller functionality. The optimal choice of trajectory profiles and execution times for point-to-point movements offers distinct energy savings. From the presented results, a number of general trajectory parametrization advices of even roughly known system characteristics can be derived. Even with little effort and without knowledge of exact system parameters, considerable energy savings are achievable.
\end{abstract}

Keywords - energy efficiency, servo inverter drive, optimal trajectory profile, energy losses

\section{Introduction}

Electrically actuated automation systems, e.g. in manufacturing and other industrial sectors, exhibit significant energy consumption. Since energy prices are continuously rising and political guidelines require improvements in efficiency to reduce $\mathrm{CO}_{2}$ emissions, industrial companies are forced to reduce energy demands.

Electromechanical drives are utilized in versatile industrial applications, e.g. robotic manipulators, lift drives, or other material handling devices. Generally, the layout of the servo drive and the choice of hardware components (e.g. motors and inverters) preferably follow the mechanical loads to be actuated (masses, inertia, external forces, etc.) and the desired movement dynamics. A basic efficiency of the system is achieved by reasonable dimensioning (i.e. by avoiding unnecessary overload capacity) and an energetically compatible selection of hardware components, since available servo motors and inverters individually offer quite good efficiency.

Often, servo drives perform point-to-point (PTP) movements that are typically commanded using standard trajectory profiles (e.g. the Double-S-Velocity profile [1]) and are often driven with maximum dynamics. However, besides an energetically efficient design of the servo drive system, also the utilized trajectory characteristics have distinct influence on the consumed grid energy, since appearing energy losses in all power transmitting elements are significantly dependent on the sequence of operating points.

In [2], a reduction of energy losses in electrical drives is achieved with the application of an optimal control problem. A comparable approach is presented in [3], where a dynamic programming technique is used to reduce resistive copper losses in servo motor windings. However, friction losses in the application's mechanics or any further source of energy dissipation in the servo drive are not considered. Furthermore, the implementation of such advanced control approaches is not state of the art in industrial applications. A selection of standard trajectory profiles is applied to different manipulators and energy demand and smoothness of jerk are evaluated in [1]. But, besides the effort, i.e. the absolute actuation torques, neither explicit consideration of different reasons for energy losses nor the possibility of energy recuperation are included.

The scope of this paper is the identification of possible improvement potential in energy demand by a proper choice of standard trajectory profiles and execution times with utilization of classical cascade control of current, velocity, and position [4]. Hereby, a wide range of different servo drive applications performing PTP tasks is addressed. The subsequent investigations deliver evidence that may be used as a guideline for proper movement parametrization of specific actuation tasks on different mechanical loads with utilization of standard hardware equipment and established path planning approaches.

The article is organized as follows. In section 2 , relevant sources of energy losses in the servo drive hardware components are discussed and classified. Then, a variety of standardized trajectory profiles is revised in section 3 and the marked differences are outlined since these are relevant for the subsequent analysis. Section 4 presents versatile simulations for different PTP movements using various profile characteristics and system parameter combinations. Finally, section 5 subsumes the results and the extracted hints and advices for proper and intuitive movement parametrization. 


\section{Electromechanical servo drive}

An electrical servo drive typically comprises a mechanical load that is actuated by an electromechanical motor (e.g. asynchronous or synchronous three phase machines). Due to the required speed and torque variable operation with high dynamics, the motor is operated by a programmable logic controller (PLC) with an inverted rectifier module consisting of IGBT-modules. The servo inverter is connected to a supply module via a DC link (see Figure 1). On the DC side, a certain electrical capacitance exists in the supply and the inverter module, and, therefore, a small energy storage capacity. The considered servo drives are capable of four quadrant operation and, consequently, recuperating electrical energy from the mechanical motion, e.g. during deceleration phases. In most applications, a brake chopper and a brake resistor are utilized to dissipate the recuperated energy. Special supply module types are capable of energy recovery to the power network but the are rarely used due to higher procurement costs.

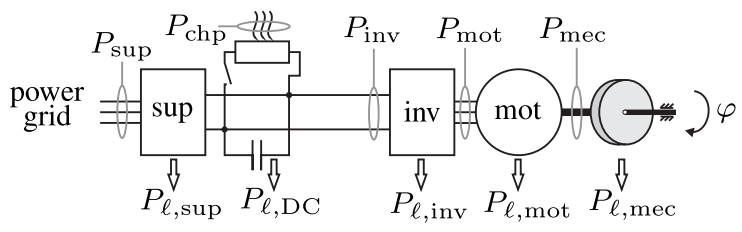

Figure 1. Connection schematic of typical single axis servo drive components

For the following investigations it is assumed that a great variety of different mechanical system loads (rotational and linear axes, spindles, etc.) that are actuated with conventional servo motors can be described using model equations for a rotational inertia [5, 6]. Hence, to fundamentally identify the energy savings potential by proper choice of trajectory profiles, rotating axes with constant mass moment of inertia, basic non-linear friction effects, and no gravitational influence or external forces are considered in this study. To cover a high quantity of different system characteristics, the mechanical parameters (e.g. inertia and friction coefficients) are recombined in different ways during the following simulations (see section 4).

\subsection{Energy balance of PTP movements}

The grid energy supply demand $E_{\text {sup }}$ needed to perform a PTP task depends on several components that are listed in the following energy balance equation. Since the mechanical axis starts and stops at rest and, therefore, with zero kinetic energy, for supply energy follows:

$$
E_{\mathrm{sup}}=E_{\ell}+E_{\mathrm{chp}}+\Delta E_{\mathrm{pot}}+\Delta E_{\mathrm{cap}},
$$

where $E_{\ell}$ are the total energy losses of the mechanics and the servo drive components, $E_{\text {chp }}$ is the energy dissipated by the brake resistor, $\Delta E_{\text {pot }}$ is the difference in potential energy before and after the movement, and $\Delta E_{\text {cap }}$ is the difference in energy storage of the servo drive capacities. For the considered servo drive axis, some simplifications can be made: the mechanical load is a symmetrical rotating inertia leading to $\Delta E_{\text {pot }}=0$. Also, the electrical capacitance in the power electronics is typically negligibly small, resulting in $\Delta E_{\text {cap }}=0$. Hence, the different amounts of energy are calculated as the time integrals of the dissipated brake chopper power $E_{\text {chp }}=\int_{t_{0}}^{t_{1}} P_{\text {chp }}(t) \mathrm{d} t$, and the total energy loss power in the mechanics and the servo drive hardware components $E_{\ell}=\int_{t_{0}}^{t_{1}} P_{\ell}(t) \mathrm{d} t$, for a PTP movement lasting from time $t_{0}$ to $t_{1}$. The total energy losses $P_{\ell}$ are (compare Figure 1):

$$
\begin{aligned}
P_{\ell}(t) & =P_{\ell, \text { sup }}(t)+P_{\ell, \mathrm{DC}}(t)+P_{\ell, \text { inv }}(t) \\
& +P_{\ell, \text { mot }}(t)+P_{\ell, \text { mec }}(t) .
\end{aligned}
$$

The different sources of energy losses of the different system components are discussed in the following section.

\subsection{Energy losses}

With selection of high-quality components and reasonable dimensioning, high efficient servo drive applications can be realized. However, besides the mechanical load, all power transmitting elements of the servo drive also produce certain energy losses during operation. Manufacturers usually name the efficiency factors $\eta_{\mathrm{N}}$ for drive operation at nominal rating. Typical values for three phase permanent magnet synchronous motors (PMSM) and power inverters are above 0.9 and 0.95 , respectively [4]. However, the efficiency distinctly deviates for different operating points, defined by the motor velocity $\dot{\varphi}(t)$ and motor torque $\tau(t)$. Therefore, the resulting energy losses variegate during the axis movement, depending on the operating point, the related electrical currents, the DC link voltage levels, the temperature, etc. The combined overall efficiency of the complete servo drive application and the calculation of the supply energy demand for given trajectories is subject of the following sections.

\subsubsection{Mechanical load}

The mechanical power $P_{\text {mec }}$ of a rotational axis with total inertia of the motor axis $J$ and transmission gears (if existing) is:

$$
P_{\mathrm{mec}}(t)=\dot{\varphi}(t)\left[J \ddot{\varphi}(t)+f_{\mathrm{v}} \dot{\varphi}(t)+f_{\mathrm{c}} \operatorname{sgn}(\dot{\varphi}(t))\right],
$$

where the friction coefficients $f_{\mathrm{v}}$ and $f_{\mathrm{c}}$ represent all dissipative effects due to viscous damping and Coulomb 
friction, respectively. Energy dissipation due to friction may arise in bearings, gear transmissions, sealing washers, oil bath lubrication, etc. As can be seen in (3), friction loss power is proportional to the drive velocity $\dot{\varphi}$ for Coulomb friction and proportional to the square of the drive velocity $\dot{\varphi}^{2}$ for viscous damping.

\subsubsection{Synchronous motor}

In the considered three-phase electromechanical motor, where the mechanical movement is provoked by the interaction of magnetic fields in the rotating armature and the stator, different effects cause dissipation of energy and, hence, have impact on the motor's efficiency. PMSMs possess a constant armature field, induced by high-performance magnets that are typically mounted on the armature's surface. The stator field on the contrary is induced using copper windings and field-orientated current control. The motor input power $P_{\text {mot }}$ is:

$$
P_{\text {mot }}(t)=P_{\text {mec }}(t)+P_{\ell, \text { mot }}(t) \text {, }
$$

where $P_{\ell \text {,mot }}$ combines all energy losses produced by the synchronous motor. A motor shaft rotation leads to continuous reversal of magnetism and, therefore, to iron losses due to magnetic hysteresis and eddy currents. Friction and windage losses in the motor are identically handled as described for the mechanical load, see section 2.2.1.

Since the motor torque $\tau(t)=k_{\mathrm{m}} i_{\mathrm{mot}}(t)$ is approximately proportional to the motor current by the motor constant $k_{\mathrm{m}}$, resistive losses in the stator windings are assumed as proportional to the square of the motor current $i_{\text {mot }}^{2}$ and, therefore, proportional to the square of motor torque $\tau^{2}$. In contrast, the hysteresis and eddy current losses are approximately proportional to the motor shaft velocity $\varphi$, and proportional to the square of the motor shaft velocity $\varphi^{2}$, respectively [7]. Further loss effects, e.g. due to harmonics, insufficient isolation, or field weakening are not considered since negligible small.

\subsubsection{Power electronics}

The total input power of the servo inverter $P_{\mathrm{inv}}$ is:

$$
P_{\text {inv }}(t)=P_{\text {mot }}(t)+P_{\ell, \text { inv }}(t)+P_{24 \mathrm{~V}}(t),
$$

where the total servo inverter losses $P_{\ell \text {,inv }}$ mainly result from resistive losses in conductor paths and switching losses in insulated gate bipolar transistors (IGBTs) during the pulse width modulated (PWM) three-phase voltage generation to feed the PMSM. Further demands are covered by a $24 \mathrm{~V}$ power supply $\left(P_{24 \mathrm{~V}}\right)$, e.g. for the PLC electronics, I/O-interface, or the optional mechanical motor holding brake.
Hence, resistive losses are considered proportional to the square of the total inverter current $i_{\text {inv }}^{2}$, which also has approximate proportionality to the motor torque $\tau$, whereas IGBT switching losses are proportional to the constant PWM switching frequency, e.g. $8 \mathrm{kHz}$. Further losses in the servo drive system basically result from resistive conduction losses in the diode rectifier of the supply module and from leakage currents in the DC link capacitors due to limited isolation. However, experiments showed that these are comparatively small and, therefore, neglected in the following. Finally, also neglecting the small DC link capacitance, the supply module input power $P_{\text {sup }}$, which represents the power consumption of the complete servo drive system, and the dissipated brake chopper power $P_{\text {chp }}$ are:

$$
\begin{aligned}
& P_{\text {sup }}(t)=P_{\text {inv }}(t) H\left[P_{\text {inv }}(t)\right], \\
& P_{\text {chp }}(t)=-P_{\text {inv }}(t) H\left[-P_{\text {inv }}(t)\right],
\end{aligned}
$$

where the Heaviside step function $H$ is defined as:

$$
H[n]=\left\{\begin{array}{ll}
0, & n<0 \\
1, & n \geq 0
\end{array} .\right.
$$

\subsection{Energy loss categorization}

Based on the preceding sections, all decisive energy losses of the utilized servo drive components are classified in three groups: constant energy losses $P_{\ell \text {,con }}$, motor speed-sensitive energy losses $P_{\ell \text {,vel }}$ and torque-sensitive energy losses $P_{\ell, \text { trq }}$. Hence, in comparison to (2), the total energy losses $P_{\ell}$ can also be expressed as:

$$
P_{\ell}(t)=P_{\ell, \mathrm{con}}(t)+P_{\ell, \mathrm{vel}}(t)+P_{\ell, \mathrm{trq}}(t) .
$$

Table 1 subsumes the mentioned dependencies. To calculate energy losses of the servo drive components properly parametrized model equations have been defined and validated (see [6]), and are utilized in the following.

\section{Trajectory profiles}

In this research, PTP movements of single axis servo drives are addressed. Hence, the axis position profile $\varphi(t)$ of a trajectory starting at time $t_{0}$ and ending at time $t_{1}$ is bounded by the following constraints:

$$
\begin{aligned}
& \varphi\left(t_{0}\right)=\varphi_{0}, \quad \varphi\left(t_{1}\right)=\varphi_{1}, \\
& \dot{\varphi}\left(t_{0}\right)=\ddot{\varphi}\left(t_{0}\right)=\dot{\varphi}\left(t_{1}\right)=\ddot{\varphi}\left(t_{1}\right)=0 .
\end{aligned}
$$

Hence, a variety of different trajectory profile types comes into consideration. To limit the number of profile variations and to comply with the requirements for practical implementation, some specifications are defined for the utilized trajectories. First, the position profile $\varphi(t)$ 
Table 1. Qualitative categorization of energy losses by proportionality to the operating point $(\dot{\varphi}, \tau)$

\begin{tabular}{|c|c|c|c|c|}
\hline & loss type & speed & torque & const. \\
\hline \multirow{2}{*}{$\begin{array}{l}\text { T. } \\
\text { O }\end{array}$} & Coulomb friction & $\sim \dot{\varphi}$ & - & - \\
\hline & viscous damping & $\sim \dot{\varphi}^{2}$ & - & - \\
\hline \multirow{6}{*}{$\begin{array}{l}\stackrel{\check{0}}{0} \\
\stackrel{0}{\Xi}\end{array}$} & resistive & - & $\sim \tau^{2}$ & - \\
\hline & hysteresis & $\sim \dot{\varphi}$ & - & - \\
\hline & eddy currents & $\sim \dot{\varphi}^{2}$ & - & - \\
\hline & Coulomb friction & $\sim \dot{\varphi}$ & - & - \\
\hline & viscous damping & $\sim \dot{\varphi}^{2}$ & - & - \\
\hline & holding brake & - & - & const. \\
\hline \multirow{3}{*}{ 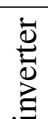 } & resistive & - & $\sim \tau^{2}$ & - \\
\hline & IGBT switching & - & - & const. \\
\hline & PLC controller & - & - & const. \\
\hline
\end{tabular}

should be continuous up to the second time derivative $\ddot{\varphi}(t)$, i.e. the acceleration. Hereby, the jerk is limited $\left(|\dddot{\varphi}(t)| \leq \dddot{\varphi}_{\lim }\right)$ to prevent the mechanics from damage and reduce wearing $[8]$.

\subsection{Trajectory profile types and categorization}

The following list describes a selection of different trajectory profiles, that comply with the above mentioned requirements:

P1) Trapezoidal acceleration profile: With utilization of the trapezoidal acceleration profile (a.k.a. DoubleS-Velocity profile), the time-optimal PTP movement is achieved, since always jerk, acceleration, or velocity limitation is exhausted during the complete movement [5, 8].

P2) Sinusoidal profile: For the sinusoidal profile trigonometric functions are used to describe the acceleration and deceleration phases and a linear polynomial for the constant velocity phase. The profile enables to specifically stimulate or suppress certain frequency bands to avoid vibrations and oscillations of the mechanical set-up.

P3) Polynomial profile: The polynomial function of degree 5 is characterized by a simple mathematical expression that can easily be parametrized for different boundary constraints (see (9)).

All the named trajectory profile types are displayed in Figure 2. Here, the individual time-optimal profiles with minimum execution times $T_{\min , i}=t_{\mathrm{P}, i}-t_{0}$ with $t_{0}=0 \mathrm{~s}$ are given for an axis position displacement of 10 revolutions, when the velocity limit or acceleration limit, or both are exceeded. As can be seen, the trapezoidal acceleration profile has the shortest trajectory time $t_{\mathrm{P} 1}$ while the sinusoidal $\left(t_{\mathrm{P} 2}\right)$ and the polynomial $\left(t_{\mathrm{P} 3}\right)$ profiles possess longer minimum-time since the dynamics limitations are not continuously exploited.

The intended trajectory profile analysis also requires the calculation of valid profiles also for specifically defined longer movement durations $T_{\mathrm{def}}>T_{\min }$. In this context, it has to be pointed out that, for the trapezoidal acceleration profile (P1) two different methods can be applied to increase the movement duration for a given axis position displacement. For the first method (P1a), the time optimal trajectory profile is generated first. Then, a scaling factor is defined by the quotient of the a defined movement duration $T_{\text {def }}$ and the minimum trajectory time $T_{\min }$ as $f_{\mathrm{s}}=T_{\text {def }} / T_{\text {min }}$. With simultaneous scaling of the profiles in dimension of time using factor $k_{\mathrm{s}}$, for the position, velocity, and acceleration profiles follows:

$$
\begin{aligned}
& \varphi_{\mathrm{T}_{\text {def }}}\left(f_{\mathrm{s}} t\right)=\varphi_{\mathrm{T}_{\min }}(t) f_{\mathrm{s}}^{0}, \\
& \dot{\varphi}_{\mathrm{T}_{\text {def }}}\left(f_{\mathrm{s}} t\right)=\dot{\varphi}_{\mathrm{T}_{\min }}(t) f_{\mathrm{s}}^{-1}, \\
& \ddot{\varphi}_{\mathrm{T}_{\text {def }}}\left(f_{\mathrm{s}} t\right)=\ddot{\varphi}_{\mathrm{T}_{\min }}(t) f_{\mathrm{s}}^{-2},
\end{aligned}
$$

where $\varphi_{\mathrm{T}_{\min }}(t)$ is the motor angle position profile for the minimum movement duration $T_{\min }$ and $\varphi_{T_{\text {def }}}(t)$ is the motor position profile for the defined movement duration $T_{\text {def. }}$. The second method (P1b) generates a trapezoidal acceleration profile maintaining maximum acceleration but, instead, reaches a lower velocity level with rising movement duration.

Figure 3 displays the four resulting trajectory types (P1a, P1b, P2, and P3) with the same position displacement of 10 axis revolutions for a predefined movement duration of $T_{\text {def }}=1 \mathrm{~s}$. Apparently, all the resulting profiles fulfill the given displacement and duration requirements but obviously provide different movement characteristics. While the trapezoidal profile (P1b) and the sinusoidal profile (P2) still reach maximum acceleration values and, therefore, have lower velocities, the scaled trapezoidal profile (P1a) and the polynomial (P3) accelerate at lower levels but instead achieve higher velocity levels.

\subsection{Expected influence on energy losses and supply}

Based on the mentioned profile characteristics highlighted in the previous section, the relationship of torque and speed-sensitive as well as constant energy losses must be analyzed for the given servo drive application. Depending on the system parameters (friction coefficients, inertia, etc.) as well as the desired position displacement, the ratio of losses may change with the choice of trajectory profile type. Hence, for specific PTP tasks of different servo drive applications, the utilization of a particular trajectory profile type as well as an optimal movement duration should lead to the most efficient movement with minimal energy losses. In this context, the following three assumptions can be concluded: 


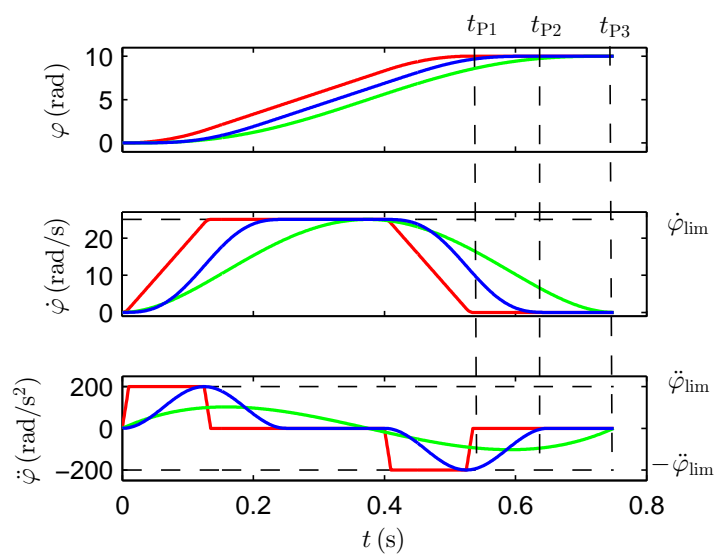

Figure 2. Trajectory profiles P1 (red), P2 (blue), and $\mathrm{P} 3$ (green) with individual minimum time durations $T_{\min , i}=\left\{t_{\mathrm{P} 1}, t_{\mathrm{P} 2}, t_{\mathrm{P} 3}\right\}-t_{0}$
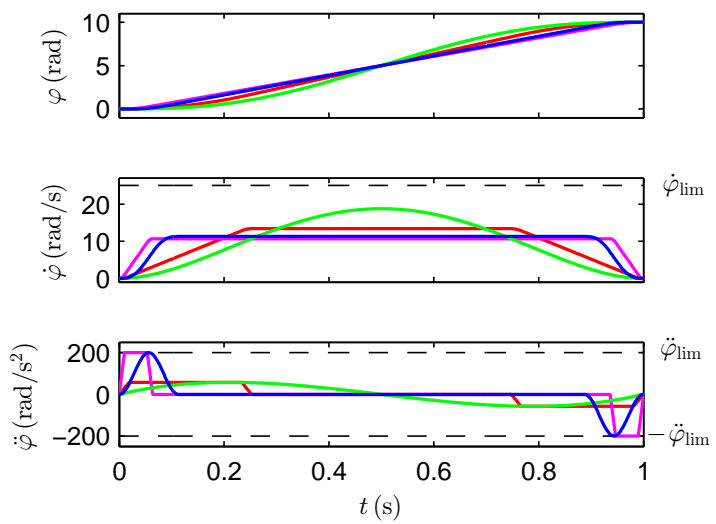

Figure 3. Trajectory profiles P1a (red), P1b (magenta), P2 (blue), and P3 (green) with a defined movement duration of $t_{1}=1 \mathrm{~s}$

1) Utilization of trajectory profiles (P1b) and (P2) leads to high torque-sensitive energy losses due to high acceleration values but moderate velocity-sensitive energy losses due to lower speed levels.

2) Utilization of trajectory profiles (P1a) and (P3) leads to high velocity-sensitive energy losses due to high speed levels but moderate torque-sensitive energy losses due to lower acceleration values.

3) Velocity (and acceleration) levels decrease with a longer movement duration and, therefore, speed and torque-sensitive losses are reduced, while constant energy demands are gaining in influence.

On the basis of the aforementioned assumptions, the existence of an energetically optimal trajectory profile type and movement duration is expected for a variety of different servo drive applications, possessing specific system parameters (e.g. friction coefficients, inertia, movement dynamics/position displacement). The examination of the listed assumptions is the objective of section 4 .

\section{Trajectory profile variation}

In the following investigations, a PTP movement with a position displacement of $\Delta \varphi=\varphi_{1}-\varphi_{0}=75 \cdot 2 \pi$ is consulted. As described in section 2 , the system model of a rotational servo drive axis with inertia as well as Coulomb and viscous friction effects is utilized. The parameters of the systems mechanics as well as the energy loss model parameters have been identified following standard parameter identification methods and verified using measurements that were presented in a previous publication [6]. The temperature level of current conductors and friction partners is assumed to be stationary in warmed up condition. The utilized system parameters and the dynamics limitations of the servo drive are collected in Table 2. The acceleration limit $\ddot{\varphi}_{\text {lim }}$ for the trajectory profile calculation is obtained from $\ddot{\varphi}_{\lim }=\tau_{\lim } / J$. Additional motor torque due to friction during the axis movement is accepted since the servo drive provides high overload capability $\tau_{\text {ult }}$.

For the given system dynamics, a minimum trajectory time of $T_{\min }=1.221 \mathrm{~s}$ results under utilization of the trapezoidal acceleration profile (P1). To simulate the energy demands for different situations, $n=20$ particular movement durations $T_{\mathrm{def}, i}$ are defined using a exponential relation:

$$
T_{\text {def }, j+1}=T_{\min } 10^{\frac{j}{n-1}}, j=\{0,1, \ldots, n-1\},
$$

leading to a maximum servo drive movement duration variation beginning from $T_{\mathrm{def}, 1}=T_{\min }=1.221 \mathrm{~s}$ up to $T_{\text {def }, 20}=10 \cdot T_{\min }=12.210 \mathrm{~s}$.

Table 2. System parameters and dynamics limits

\begin{tabular}{llr}
\hline parameter & symbol & value \\
\hline inertia & $J\left(\mathrm{~kg} \mathrm{~m}^{2}\right)$ & 0.011 \\
Coulomb friction & $f_{\mathrm{c}}(\mathrm{Nm})$ & 0.100 \\
viscous damping & $f_{\mathrm{v}}\left(\frac{\mathrm{Nms}}{\mathrm{rad}}\right)$ & 0.002 \\
\hline speed limit & $\dot{\varphi}_{\lim }\left(\frac{\mathrm{rad}}{\mathrm{s}}\right)$ & 628.32 \\
torque limit & $\tau_{\lim }(\mathrm{Nm})$ & 20.00 \\
ultimate torque & $\tau_{\mathrm{ult}}(\mathrm{Nm})$ & 30.00 \\
\hline
\end{tabular}

The resulting velocity profiles of the four different profile variants for three exemplary movement durations ( $T_{\text {def, } 1}, T_{\text {def,10, }}$ and $\left.T_{\text {def,20 }}\right)$ are displayed in Figure 4. Note that the polynomial (P3) and the sinusoidal profiles (P2) for fast movements $\left(T_{\mathrm{def}, 1} \approx T_{\min }\right)$ violate the velocity and/or the acceleration limits and, therefore, are excluded. Also note that the trapezoidal acceleration profiles (P1a and P1b) are identical for $T_{\mathrm{def}, 1}=T_{\min }$. 


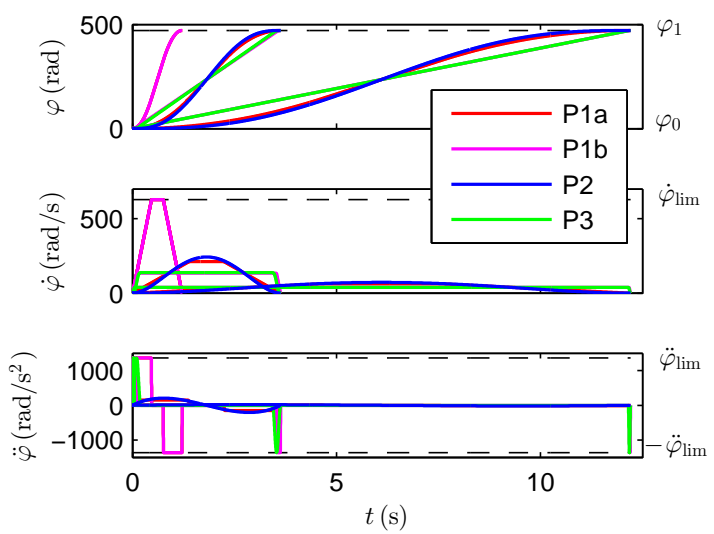

Figure 4. Different velocity profiles for three exemplary movement durations $\left(T_{\mathrm{def}, 1}, T_{\mathrm{def}, 10}\right.$, and $\left.T_{\text {def,20 }}\right)$

The following sections present simulation results showing the influence of the trajectory profile choice and the movement duration on the servo drive system's supply energy demand. Therefore, in section 4.1, the different trajectory profile variations are applied to the validated virtual rotational axis using a variation of movement durations $T_{\mathrm{def}, i}$ as defined in (11). To motivate the transferability of the presented results, additional variations of other system parameters, e.g. the friction coefficients, the inertia, and the movement dynamics (by variation of the axis displacement) are presented in section 4.2.

\subsection{Results for validated test rig model functions}

According to the specifications defined in section 4, the trajectory profile types are generated for increasing movement durations for a fixed position displacement of 75 axis revolutions. Figure 5 shows the corresponding supply energy demand values. In addition, the absolute amounts of energy losses are given, separated for different causes (compare section 2.3): constant loss power, speed and torque-specific losses.

As can be seen in the upper plot, obvious differences in energy demand exist depending on the chosen profile type and the applied movement duration. For all utilized profile types, the energy demand possesses a minimum between the movement times from 4.6 to $5.2 \mathrm{~s}$, which is approximately $\{3.77 \ldots 4.26\} \cdot T_{\min }$. In the lower plot, the different sources of energy losses are given for all trajectories. Obviously, the high energy demands of fast manipulator movements are mainly caused by high torquespecific losses, induced by high currents in the conductor resistances of the servo drive system (compare Table 1). However, with increasing movement duration, the relation between torque and speed-specific losses changes and the
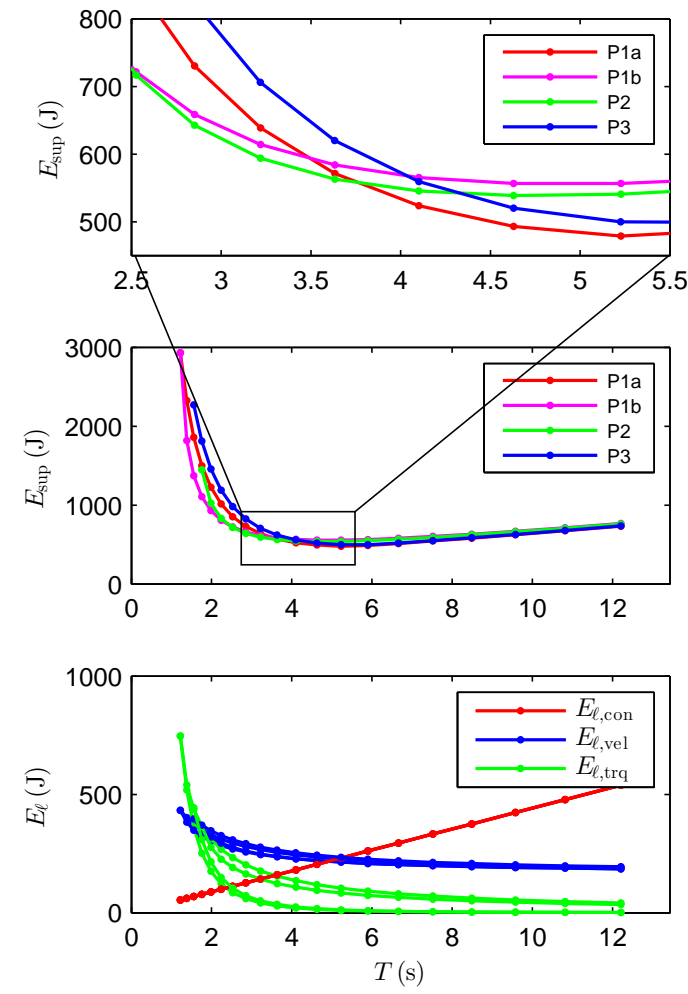

Figure 5. Total energy supply demand for different profile types and movement durations; different sources energy losses, separated for constant $\left(E_{\ell, \text { con }}\right)$ as well as velocity $\left(E_{\ell, \text { vel }}\right)$ and torque dependency $\left(E_{\ell, \text { trq }}\right)$

speed-specific losses become dominant. With a view to the upper plot, the change in loss dominance, together with an increasing influence of constant energy losses, also involves a change of energetically optimal group of trajectory profile. Apparently, for faster movements the group of trajectory profiles (P1b and P2) are more efficient while for slower movements, the other group (P1a and P3) becomes favorable. Finally, the energy demand of the utilized profiles increases again with longer execution time due to the linearly rising influence of constant power demands (of electronics, brakes, etc.). The absolute minimum energy demand for the given PTP task and the rotational axis is achieved with a movement duration of $T_{\text {def }} \approx 4.26 \cdot T_{\min } \approx 5.2 \mathrm{~s}$ and utilization of the scaled trapezoidal acceleration profile (P1a), closely followed by the polynomial (P3).

\subsection{Variation of system parameters}

For the motivation of general statements about an advantageous choice of the profile type and the optimal 
movement duration for a favorably wide range of different servo drive applications, the same calculations are repeated for a set of parameter variations. Therefore, the Coulomb friction and viscous damping coefficients $f_{\mathrm{c}}$ and $f_{\mathrm{v}}$ are incrementally increased using a factor $f=\{1, \ldots, 10\}$ in 20 equidistant steps, based on the validated system model parameters utilized in section 4.1. In this manner, the same energy demand analysis is accomplished for similar servo drive axes, that possess higher friction influence. The results are presented and discussed in section 4.2.1. Furthermore, section 4.2.2 presents the energy demand results for increased inertia of the mechanical load. Finally, a variation of movement dynamics is consulted in section 4.2 .3 by increasing the desired position displacement under retention of the movement durations $T_{\mathrm{def}, i}$ defined in (11) at the beginning of this section.

\subsubsection{Variation of friction coefficients}

In comparison to the lower plot in Figure 5, the surface plots in Figure 6 present the proportion between constant, speed and torque-specific losses also with a variation of the system's friction coefficients. As expected, the velocity dependent losses gain in importance with increasing friction parameters, while the torque-specific relations remain more or less untouched.

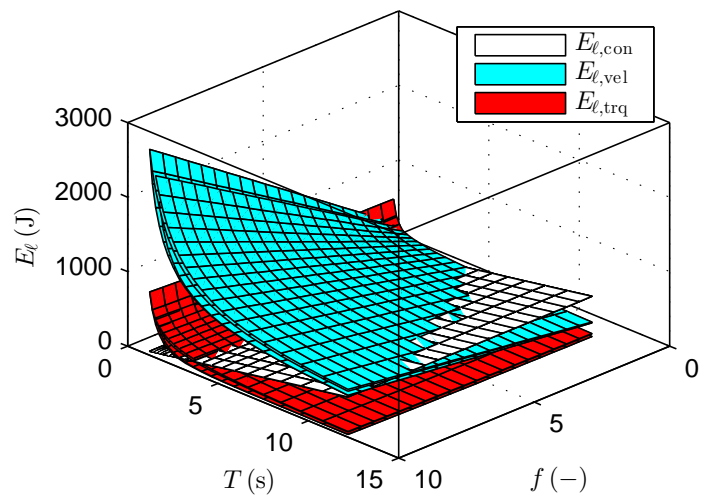

Figure 6. Energy losses, separated for constant $\left(E_{\ell, \mathrm{con}}\right)$ as well as velocity- $\left(E_{\ell, \mathrm{vel}}\right)$ and torquedependency $\left(E_{\ell, \text { trq }}\right)$

As shown in Figure 7, for fast servo drive movements, the trapezoidal acceleration profile with maximum acceleration (P1b) (and for a smaller range the sinusoidal profile (P2)) demands the lowest energy supply (independent from the friction intensity). For systems with low friction influence $(f \leq 7)$, the energetically optimal profile selection switches to the group of profile types $\mathrm{P} 1 \mathrm{a}$ and $\mathrm{P} 3$ (here, to the scaled trapezoidal acceleration profile P1a) after an approximate movement duration of $T_{\text {def }, i} \approx 2 \cdot T_{\min } \approx 2.5 \mathrm{~s}$, since lower acceleration levels reduce the torque-specific energy losses. The yellow line highlights the optimal movement duration which increases with the friction variation.

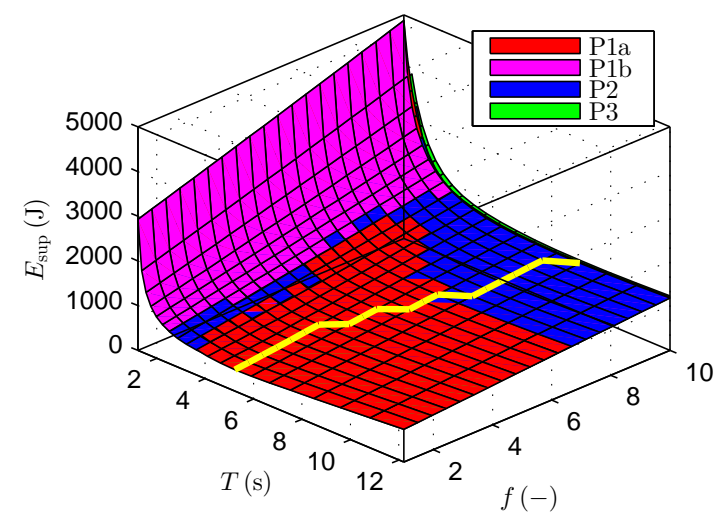

Figure 7. Supply energy demand $E_{\text {sup }}$ for different profile types, movement durations $T$ and friction coefficient variation by factor $f$ (bottom view)

Since the surface plots of the supply energy results are relatively close, Figure 8 presents the relative difference in supply energy, to emphasize the possible savings potential by adequate choice of the trajectory profile. The results show that especially for fast PTP movements of the servo drive a high relative difference in supply energy demand exists of about 20 to $40 \%$. For slower movements a relative difference of 5 to $10 \%$ remains, which also illustrates the high importance of a reasonable trajectory profile choice.

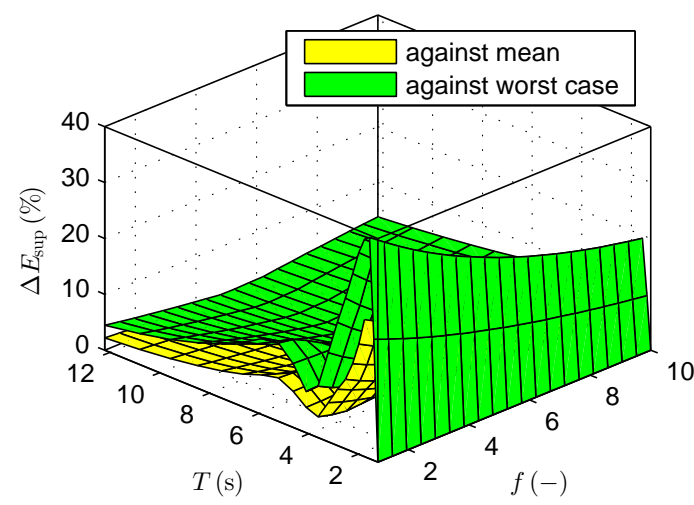

Figure 8. Relative difference in supply energy demand between the most efficient against the mean value and against the worst case 


\subsubsection{Variation of mechanical inertia}

Figure 9 shows the supply energy demand results $E_{\text {sup }}$ for a variation of the mechanical inertia using a factor $f=\{1 \ldots 2\}$ in 20 equidistant discrete steps. For higher inertia many of the profile types reach the torque ultimate limits. As shown in the plot for fast axis movements, the same trajectory profiles, i.e. profile $\mathrm{P} 1 \mathrm{~b}$ followed by profile $\mathrm{P} 2$ are the most efficient choice. A change of the best profile with increased inertia parameter does not appear, since compared to the increased friction effects in section 4.2.1, the higher inertia leads to increasing torquespecific energy losses, which are smaller for trajectory profile group including types P1a and P3. Again, the optimal movement duration increases slightly with higher inertia, marked by the yellow line. The resulting values of relative energy demand show similar savings potential.

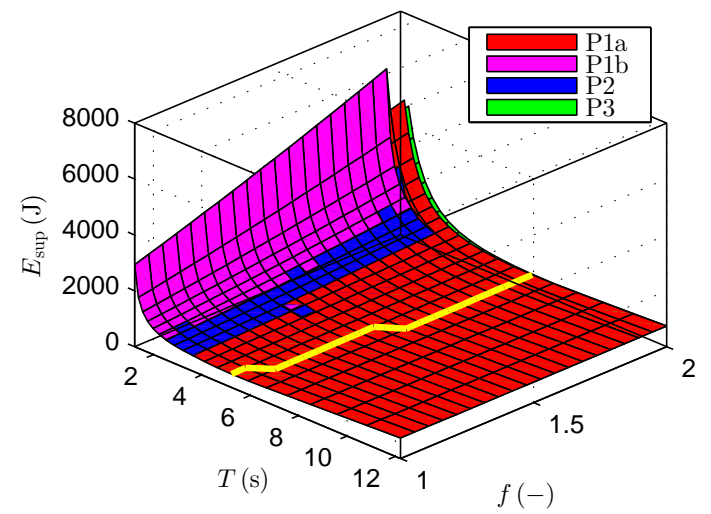

Figure 9. Supply energy demand $E_{\text {sup }}$ for different profile types, movement durations $T$ and variation of mechanical inertia by factor $f$ (bottom view)

\subsubsection{Variation of axis dynamics}

The last simulation example presents the supply energy demand results and corresponding optimal trajectory profile types for an increasing axis displacement for the given variation of movement durations (see Figure 10). Since the values of minimum movement time increases with the desired displacement $\Delta \varphi$, the a rising number invalid trajectories for small times $T_{\text {def }, i}$ appears with increasing displacement. The factor $f=\{1 \ldots 3\}$ is incrementally increased in 20 steps. Rising axis displacement leads to higher velocity levels and longer acceleration phases, to cover the longer distances within the given movement duration. This leads to both, increased speed and torquespecific energy losses, while the constant losses remain on their ratio. The resulting map of optimal trajectory profiles is similar to the previous one (compare section 4.2.2).
After a certain raise of defined movement duration related to the minimum movement times of each axis displacement variation, the optimal profile type switches from $\mathrm{P} 1 \mathrm{~b}$ to P2 and finally to P1a.

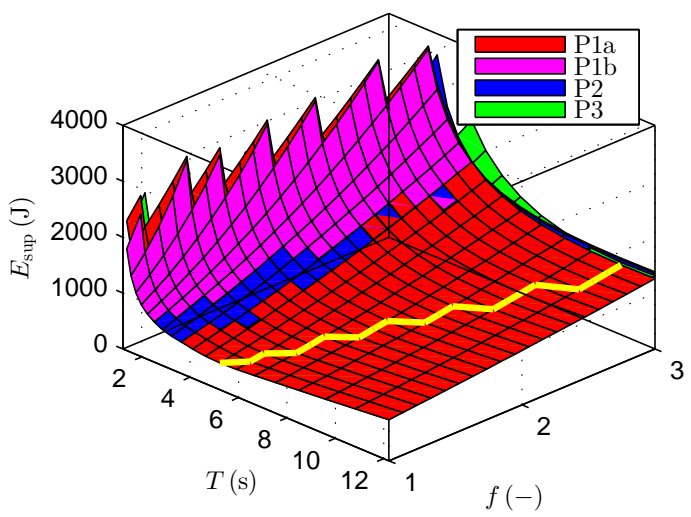

Figure 10 . Supply energy demand $E_{\text {sup }}$ for different profile types, movement durations $T$ and variation of movement displacement by factor $f$ (bottom view)

\subsection{Discussion of Results}

Although during the presented simulation, different system parameters are variegated, comparable trends become apparent. As can be seen in Figures 7, 9, and 10, for fast PTP movements (close to $T_{\min }$ ) of the given rotational servo drive axis, the most efficient trajectory is the trapezoidal acceleration profile (P1b). However, for all test series, the optimal profile type switches to the sinusoidal profile (P2) after an approximate extension of the movement duration by factor $2\left(T_{\text {def }} \approx 2 \cdot T_{\min }\right)$. With slower movements, the energetically optimal trajectory profile finally switches to the scaled acceleration profile (P1a), with exception of parameter configurations with higher friction influence. Here, the dominant source of energy loss changes, resulting in a preference of profiles, that achieve lower velocity levels. The polynomial trajectory profile only offers best system efficiency in exceptional cases (not shown).

Furthermore, there exists a clear trend for the energetically optimal movement duration that is similar for all parameter configurations. For all test series, the optimal movement duration for the given PTP task (including the increased position displacement in section 4.2.3) appears in a range between $T_{\mathrm{def}}=\{3.5 \ldots 4.5\} \cdot T_{\min }$. Of course, the factor is dependent on the amount of constant power demands effecting the raise of energy supply for longer movement duration. In all these cases, the optimal profile type was the scaled trapezoidal acceleration profile (P1a). 


\section{Conclusion}

The influence of different standard (jerk bounded) trajectory profiles for PTP tasks on the total supply energy demand of servo drive applications has been presented. Since with different trajectory profiles, the servo drive's operating points (motor speeds and torques) during the movement change, different causes of energy losses are evoked to a greater or lesser extent. Furthermore, the influence of constant energy losses on the complete system's energy balance must not be neglected. Based on a validated system model parametrization for a rotational servo drive axis, different parameters have been adapted for variable friction coefficients, load inertia, and movement distances.

The results show the significant difference in total energy demand of a servo drive while changing the motion profiles and the movement duration within reasonable limits. Due to the influence of constant energy demands, there exists an energetically optimal movement duration which is different from minimum time.

During the parameter variation series, repetitious characteristics became apparent, resulting in a number of general advices for the choice of trajectory profile. For all examined parameter series, a constant order of favorable profile types turned out as energetically optimal with increasing movement duration, only with exception of the case where a chance of the dominant energy loss category appears. Here, a deliberate choice of trajectory profile is recommended. Generally, already slightly increased execution times lead to the energetically optimal movement with considerable savings and, hence, to efficiency improvement.

Future works will focus on the examination of additional parameter variations and the combination of different parameters to variate. Furthermore, a wider range of different servo drive applications must be investigated, e.g. by the inclusion of different non-linear system mechanics and the influence of external forces.

\section{Acknowledgment}

The authors gratefully acknowledge support by research grants from the German Research Foundation (DFG; OR 196/4-2) and reviewers' comments.

\section{References}

[1] Boscariol, P., Gasparella, A., Gasparetto, A., Lever, N., Richiedei, D., Trevisani, A., and Vidoni, R. Energy efficiency and smoothness in robotics trajectory planning: numerical simulation and comparison. In Proc. of the Austrian Robotics Workshop, pages 7-12, 2013.

[2] Botan, C., Ratoi, M., Ostafi, F., and Horga, V. Minimum Energy Control of Servo Drive Systems with PMSM. In Proc. of the Int. Symp. on Power Electronics, Electrical Drives, Automation and Motion, pages 19-23, 2010.

[3] Klenke, F. and Hofmann, W. Energy-efficient control of induction motor servo drives with optimized motion and flux trajectories. In Proc. of the 2011-14th European Conf. on Power Electronics and Applications, pages 1-7, 2011.

[4] Kiel, E. Drive Solutions: Mechatronics for Production and Logistics. Springer, 2008.

[5] Hansen, C., Kotlarski, J., and Ortmaier, T. Path Planning Approach for the Amplification of Electrical Energy Exchange in Multi Axis Robotic Systems. In Proc. of the 2013 IEEE Int. Conf. on Mechatronics and Automation, pages 44-50, 2013.

[6] Hansen, C., Kotlarski, J., and Ortmaier, T. Experimental Validation of Advanced Minimum Energy Robot Trajectory Optimization. In Proc. of the 2013 IEEE Int. Conf. on Advanced Robotics, 2013.

[7] Wach, W. Dynamics and Control of Electrical Drives. Springer, 2011.

[8] Biagiotti, L. and Melchiorri, C. Trajectory Planning for Automatic Machines and Robots. Springer, 2008. 